PROCEEDINGS OF THE

AMERICAN MATHEMATICAL SOCIETY

Volume 30, No. 1, September 1971

\title{
SOME RADICAL PROPERTIES OF $s$-RINGS
}

\author{
MICHAEL RICH
}

ABSTRACT. The class of $s$-rings includes as a proper subset the classes of associative, alternative, Lie, Jordan, and standard rings. It is shown that in any $s$-ring $R$ the prime radical of $R$ coincides with the Baer lower radical of $R$. Relationships between the prime radical and certain other radicals are also given.

In [6], P. Zwier defines an $s$-ring $R$ to be a nonassociative ring satisfying the condition that if $A$ is an ideal of $R$ then $A^{s}$ is also an ideal of $R$, where $s$ is an integer $\geqq 2$. Also an ideal $P$ of an $s$-ring $R$ is called a prime ideal if whenever $A_{1} A_{2} \cdots A_{s} \subseteq P$ for ideals $A_{i}$ of $R$ then $A_{j} \subseteq P$ for some $j$. Here $A_{1} A_{2} \cdots A_{8}$ denotes the product of the ideals under all possible associations. It is announced that using these definitions one may define a prime radical and obtain results for $s$ rings corresponding to the results obtained by C. Tsai [5] for Jordan rings.

Specifically, if one defines the prime radical $\beta(R)$ of an $s$-ring $R$ so that it coincides with the intersection of all prime ideals of the ring $R$ and a ring $R$ to be $\beta$-semisimple if $\beta(R)=(0)$ then one obtains the following results for any $s$-ring $R$ :

1. $\beta(R) \subseteq N(R)$ where $N(R)$ denotes the nil radical of $R$.

2. $R / \beta(R)$ is $\beta$-semisimple.

3. $R$ is $\beta$-semisimple if and only if $R$ contains no nonzero nilpotent ideals.

In this note we consider the Baer lower radical of an $s$-ring $R$ and show that, just as in the case of associative rings, it coincides with the prime radical. In addition we show relationships between the prime radical, a Jacobson radical studied by Brown and McCoy and a radical of Smiley.

Recall that the Baer lower radical of a ring $R$ is defined as follows: Let $N_{0}$ be the union of all nilpotent ideals of $R$. Let $N_{1}$ be the union of all ideals $A$ in $R$ such that $A / N_{0}$ is nilpotent in $R / N_{0}$. In general if $\beta$ is a limit ordinal then $N_{\beta}=\bigcup_{\alpha<\beta} N_{\alpha}$. If $\beta$ has a predecessor then $N_{\beta}$ is the union of all ideals $A$ in $R$ such that $A / N_{\beta-1}$ is nilpotent in $R / N_{\beta-1}$. If $\gamma$ is the least ordinal such that $N_{\gamma}=N_{\gamma+1}=\cdots$ then $N_{\gamma}$

Received by the editors June 8, 1970.

AMS 1969 subject classifications. Primary 1710.

Key words and phrases. s-ring, prime ideal, nilpotent ideal, prime radical, Baer lower radical, Jacobson radical, semisimple.

Copyright (c) 1971, American Mathematical Society 
is called the Baer lower radical of $R$ and is denoted by $B(R)$. By the definition it is clear that $R / B(R)$ has no nonzero nilpotent ideals.

THEOREM 1. In any s-ring $R, B(R)$ is the intersection of all ideals $Q_{i}$ of $R$ such that $R / Q_{i}$ has no nonzero nilpotent ideals.

Proof. We may adopt the proof of this same theorem for associative rings as presented in $[2$, p. 56] since associativity is not required for that argument.

THEOREM 2. In any s-ring $R, \beta(R)=B(R)$.

Proof. Let $P$ be a prime ideal of $R$. Then $(\overline{0})$ is a prime ideal of $\bar{R}=R / P$ and $R / P$ is $\beta$-semisimple. Therefore, by property $3, R / P$ has no nonzero nilpotent ideals. Thus $P=Q_{i}$ for some $i$ and the intersection of all prime ideals in $R$ contains $W=\cap Q_{i}$. Therefore $\beta(R)$ $\supseteq B(R)$.

Conversely, suppose that $a \notin B(R)$. Clearly $(a)^{*} \nsubseteq B(R)$ for if $(a)^{*}$ $\subseteq B(R)$ then $(a) / B(R)$ is a nonzero nilpotent ideal of $R / B(R)$ which is impossible. Construct the set $A=\left\{a_{1}, a_{2}, \cdots, a_{n}, \cdots\right\}$ as follows: $a_{1}=a, a_{2} \in\left(a_{1}\right)^{\circ} \cap C(B(R)), \cdots, a_{n+1} \in\left(a_{n}\right)^{\circ} \cap C(B(R))$ where $C$ denotes set theoretic complement. By our choice of $A, A \cap B(R)=\varnothing$. Now by Zorn's lemma the family of all ideals of $R$ which are disjoint from $A$ contains a maximal ideal $P$. We show that $P$ is a prime ideal.

Let $P_{1}, P_{2}, \cdots, P_{s}$ be ideals of $R$ such that $P_{1} P_{2} \ldots P_{s} \subseteq P$. If $P_{i} \nsubseteq P$ for every $i$ then $\left(P_{i}+P\right) \Phi P$ for every $i$. Therefore $\left(P_{i}+P\right) \cap A$ $\neq \varnothing$ for every $i$ and there exist $a_{i_{1}} \in P+P_{1}, a_{i_{2}} \in P+P_{2}, \cdots, a_{i_{6}} \in P$ $+P_{s}$. Hence $\left(a_{i_{1}}\right)\left(a_{i_{2}}\right) \cdots\left(a_{i_{s}}\right) \subseteq\left(P+P_{1}\right)\left(P+P_{2}\right) \cdots\left(P+P_{s}\right)$ $\subseteq P_{1} P_{2} \cdots P_{s}+P \subseteq P$. (Here $(a)$ denotes the principal ideal generated by $a$.) By our choice of the $a_{i_{j}},\left(a_{n+1}\right) \subseteq\left(a_{n}\right)$. Let

$$
m=\max \left(i_{1}, i_{2}, \cdots, i_{s}\right) .
$$

Then $a_{m+1} \in\left(a_{m}\right)^{s} \subseteq\left(a_{i_{1}}\right)\left(a_{i_{2}}\right) \cdots\left(a_{i_{s}}\right) \subseteq P$. Thus $a_{m+1} \in P$ which contradicts the fact that $A \cap P=\varnothing$. Therefore $P$ is a prime ideal, $a \notin P$ and $\beta(R) \subseteq B(R)$.

Brown and McCoy have defined the Jacobson radical $J(R)$ of a nonassociative ring $R$ to be the maximal quasi-regular ideal of $R$ where $a \in R$ is said to be quasi-regular if $a \in Q(a)$ with $Q(a)$ the right ideal generated by $a t-t$ with $t \in R$. They have shown (see [1, Theorem 8]) that $J(R)$ is $v$-prime for any $v$ and that $N(R) \subseteq J(R)$. Thus $J(R)$ is a prime ideal according to the definition given here. Therefore $\beta(R) \subseteq J(R)$. Smiley [4] has provided a radical $S(R)$ which is the intersection of all modular maximal ideals of $R$. It is known [1, p. 254 ] that $J(R) \subseteq S(R)$. Therefore, for any $s$-ring we have the following: 
THEOREM 3. In any s-ring $R, B(R)=\beta(R) \subseteq N(R) \subseteq J(R) \subseteq S(R)$.

Finally, it should be noted that the class of $s$-rings includes associative, alternative, Lie, Jordan and standard rings. The first three can very easily be shown to be 2-rings and the latter two are 3-rings as shown in [5, Lemma 3] and [3, Lemma 1].

\section{BIBLIOGRAPHY}

1. B. Brown and N. McCoy, Prime ideals in nonassociative rings, Trans. Amer. Math. Soc. 89 (1958), 245-255. MR 20 \#3196.

2. N. Divinsky, Rings and radicals, Univ. of Toronto Press, Toronto, Canada, 1954.

3. R. D. Schafer, Standard algebras, Pacific J. Math. 29 (1969), 203-223. MR 39 $\# 5647$.

4. M. F. Smiley, Application of a radical of Brown and McCoy to non-associative rings, Amer. J. Math. 72 (1950), 93-100. MR 11, 311.

5. C. E. Tsai, The prime radical in a Jordan ring, Proc. Amer. Math. Soc. 19 (1968), 1171-1175.

6. P. J. Zwier, Prime ideals and the prime radical in a class of narings, Notices Amer. Math. Soc. 16 (1969), 660. Abstract \#69T-A89.

Temple University, Philadelphia, Pennsylvania 19122 